\title{
Self-Tuning of a Robust Fuzzy PI-PD Controller
}

K.Ram charan
P. Ananth Babu

\begin{abstract}
In this paper, a simple and effective scheme for tuning of fuzzy PI-PD controller based on fuzzy logic is proposed. Here the input scaling factors are tuned online by gain updating factors where values are determined by rule base with error and change in error as inputs according to the required controlled process. The performance comparison of conventional fuzzy logic controller, autotuned fuzzy controller with cross auto-tuned fuzzy PI-PD controllers has been done in terms of transient state and steady state. Simulation results show the effectiveness and robustness of the proposed tuning mechanism.
\end{abstract}

The full text of the article is not available in the cache. Kindly refer the IJCA digital library at www.ijcaonline.org for the complete article. In case, you face problems while downloading the full-text, please send a mail to editor at editor@ijcaonline.org 